\title{
Distribuição geográfica dos hospedeiros intermediários do Schistosoma mansoni nos estados do Paraná, Minas Gerais, Bahia, Pernambuco e Rio Grande do Norte, 2012-2014*
}

\author{
doi: $10.5123 /$ S1679-49742018000300016
}

\author{
Geographical distribution of intermediate hosts of Schistosoma mansoni in the states of \\ Paraná, Minas Gerais, Bahia, Pernambuco and Rio Grande do Norte, Brazil, 2012-2014
}

Distribución geográfica de los huéspedes intermedios de Schistosoma mansoni en los estados
de Paraná, Minas Gerais, Bahia, Pernambuco y Rio Grande do Norte, Brasil, 2012-2014

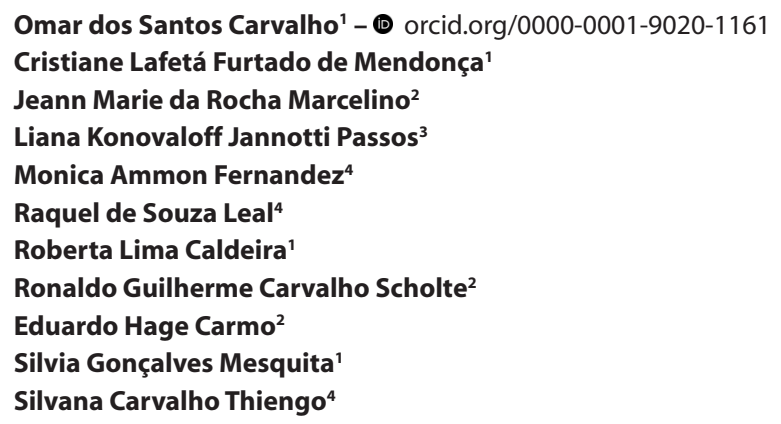

\section{Resumo}

Objetivo: descrever a distribuição geográfica dos hospedeiros intermediários do Schistosoma mansoni em cinco estados brasileiros. Métodos: estudo transversal; foram selecionados municípios dos estados do Paraná (78), Minas Gerais (120), Bahia (82), Pernambuco (51) e Rio Grande do Norte (98), nos anos de 2012 a 2014; esses municípios foram escolhidos por não possuírem registros atualizados da presença de caramujos hospedeiros intermediários de $S$. mansoni; moluscos foram capturados, taxonomicamente identificados e examinados para verificação de cercárias de $S$. mansoni . Resultados: os trabalhos foram realizados em 427 municípios (99,5\% dos 429 selecionados); foi registrada presença de moluscos em 300 (70,2\%) municípios e a ocorrência de Biomphalaria glabrata em 62 (21\%) municípios, B. straminea em 181 (60\%), B. tenagophila em três (1\%); associação de B. glabrata/B. straminea foi encontrada em 53 (18\%), e de B. glabrata/B. tenagophila em um $(0,3 \%)$. Conclusão: os registros de B. glabrata, B. straminea e B. tenagophila estão de acordo com a distribuição conhecida.

Palavras-chave: Esquistossomose; Biomphalaria; Vetores de Doenças; Mapeamento Geográfico.

\footnotetext{
*Este projeto foi financiado pela Secretaria de Vigilância em Saúde do Ministério da Saúde (Fundação Nacional de Saúde/ Ministério da Saúde:Termo de Cooperação n 173/2010), Instituto René Rachou/Fundação Oswaldo Cruz/Belo Horizonte, MG, e Instituto Oswaldo Cruz/Rio de Janeiro, RJ, desenvolvido em conformidade com o art. 14 da Lei no 5.197 , de 3 de janeiro de 1967 ('Lei da Fauna'), que dispõe sobre a concessão, a cientistas pertencentes a instituições científicas, de licença especial para a coleta de material destinado a fins científicos.
}

Endereço para correspondência:

Omar dos Santos Carvalho - Fundação Oswaldo Cruz, Instituto René Rachou, Laboratório de Helmintologia e Malacologia

Médica, Av. Augusto de Lima, n 1715, Barro Preto, Belo Horizonte, MG, Brasil. CEP: 30190-009

E-mail:omar@minas.fiocruz.br 


\section{Introdução}

A esquistossomose mansoni é uma doença infectoparasitária ${ }^{1}$ cujo agente etiológico é o trematódeo Schistosoma mansoni. Ela é endêmica em aproximadamente 49 países das Américas, Antilhas e África. No Brasil, no período de 1975 a 2012, o percentual de positividade para esquistossomose variou de 4,5 a 23,3\%, enquanto as maiores prevalências são encontradas nas regiões Nordeste e Sudeste do país. De 1988 a 2013, a taxa de internação variou de 2,5 a $0,8 / 100.000$ habitantes e a taxa de mortalidade no período de 1987 a 2012 variou de 0,5 a $0,2 / 100.000 .{ }^{1}$

\section{Biomphalaria straminea apresenta a mais ampla distribuição geográfica, estando presente em quase todas as bacias hidrográficas brasileiras. Em decorrência de sua extensa distribuição, B. straminea é a espécie mais bem adaptada a todas as variedades de climas e condições ecológicas do país.}

Os hospedeiros invertebrados estão representados por moluscos planorbídeos do gênero Biomphalaria. Entre as 11 espécies e uma subespécie de moluscos desse gênero reconhecidas no Brasil, somente Biomphalaria glabrata, Biomphalaria tenagophila e Biomphalaria straminea foram, até o momento, encontradas naturalmente infectadas pelo $S$. mansoni. 0 hospedeiro intermediário mais importante é $B$. glabrata, em função da alta suscetibilidade ao $S$. mansoni e ampla distribuição, sendo que, no Brasil, sua ocorrência está quase sempre associada à presença da esquistossomose mansoni. ${ }^{2}$

Biomphalaria tenagophila distribui-se pela faixa litorânea do sul do estado da Bahia até o Rio Grande do Sul. Nos estados de Minas Gerais, São Paulo, Paraná e Rio Grande do Sul, a espécie avança em direção ao Oeste. B. tenagophila tem importância epidemiológica no Vale do Paraíba, estado de São Paulo.

Biomphalaria straminea apresenta a mais ampla distribuição geográfica, estando presente em quase todas as bacias hidrográficas brasileiras. Em decorrência de sua extensa distribuição, $B$. straminea é a espécie mais bem adaptada a todas as variedades de climas e condições ecológicas do país. Essa espécie tem importância na epidemiologia da esquistossomose em alguns estados do Nordeste. A falta de pesquisas malacológicas no Brasil explica a escassez de dados de distribuição geográfica dessas espécies de moluscos. ${ }^{2}$

Outras três espécies, Biomphalaria peregrina, Biomphalaria amazonica e Biomphalaria cousini, e uma híbrida das duas últimas, foram infectadas experimentalmente e são consideradas hospedeiras em potencial do parasito. ${ }^{3-5}$

Em 2008, o Ministério da Saúde, seguindo recomendação do Comitê Técnico Assessor do Programa de Esquistossomose, redefiniu os dados de distribuição geográfica dos moluscos hospedeiros de $S$. mansoni nos estados, com foco de transmissão como tema de relevância para o Programa de Vigilância e Controle da Esquistossomose.

A distribuição geográfica dos hospedeiros intermediários do $S$. mansoni pelos municípios brasileiros vem sendo progressivamente atualizada, com base nos novos registros da presença das três espécies hospedeiras. ${ }^{6-24} 0$ registro de ocorrência de $B$. glabrata está delimitado pelos paralelos $0^{\circ} 53$ 'S (Quatipuru, PA), $29^{\circ} 51^{\prime} \mathrm{S}$ (Esteio, RS), 53⒋'S (Toledo, PR) e linha costeira; B. straminea, pelos paralelos $02^{\circ} 54^{\prime} \mathrm{S}$ e $31^{\circ} 00$ 'S, meridiano $44^{\circ} 43^{\prime} \mathrm{W}$ e litoral; $\mathrm{e}$ B. tenagophila, pelo quadrante delimitado entre os paralelos $10^{\circ} 12^{\prime} \mathrm{S}$ e $33^{\circ} 41^{\prime} \mathrm{S}$, meridiano $57^{\circ} 05^{\prime} \mathrm{W}$ e linha litorânea..$^{9,12}$

0 presente trabalho teve como objetivo descrever a distribuição geográfica dos hospedeiros intermediários do $S$. mansoni em cinco estados brasileiros.

\section{Métodos}

Trata-se de um estudo ecológico descritivo, realizado no período de setembro de 2012 a outubro de 2014. Foram utilizadas as técnicas de levantamento quantitativo e qualitativo (identificação morfológica) de moluscos hospedeiros intermediários do $S$. mansoni e informações previamente cadastradas no acervo da coleção de moluscos do Instituto Oswaldo Cruz, Fundação Oswaldo Cruz, Rio de Janeiro, RJ (Fiocruz/CMIOC). A área de abrangência do estudo compreendeu 429 municípios nos estados do Paraná (78), Minas Gerais (120), Bahia (82), Pernambuco (51) e Rio Grande do Norte (98). Esses municípios foram escolhidos por não possuírem registros da presença de caramujos hospedeiros intermediários de $S$. mansoni. As coletas foram realizadas por equipe composta de pesquisadores e profissionais 
dos municípios, previamente capacitados nos laboratórios de referência responsáveis por cada estado.

Adotou-se 0 critério de trabalhar três coleções hídricas por município selecionado, priorizando-se aquelas onde as populações locais têm maior contato com água e onde há condições favoráveis à ocorrência de planorbídeos. ${ }^{18}$

O levantamento dos moluscos foi realizado em diferentes pontos das coleções hídricas. Foi utilizada concha de metal perfurada (furos em torno de $2 \mathrm{~mm}$ ) ou peneira acoplada a um cabo de aproximadamente $1 \mathrm{~m}$ de comprimento. Raspou-se a vegetação submersa às margens e o fundo das coleções hídricas, para coleta de uma quantidade adequada de exemplares. Nesse tipo de levantamento, não há técnica amostral definida. Todos os exemplares encontrados foram armazenados em recipientes plásticos, etiquetados e levados para laboratório.

Os dados das coordenadas geográficas de todas as localidades pesquisadas foram obtidos por meio de aparelhos receptores de GPS e registrados no sistema decimal.

A pesquisa de larvas de $S$. mansoni nos exemplares de molus $\cos ^{24}$ foi realizada no Laboratório de Helmintologia e Malacologia Médica do Instituto René Rachou/ Fundação Oswaldo Cruz (IRR/Fiocruz) e no Laboratório de Malacologia do Instituto Oswaldo Cruz/Fundação Oswaldo Cruz (IOC/Fiocruz). Empregou-se a técnica de estímulo luminoso ou esmagamento entre placas de vidro, para detecção da presença ou ausência de trematódeo. Nos mesmos laboratórios, além do laboratório da Secretaria de Estado da Saúde do Paraná, foram também realizadas as identificações morfológicas.
A dissecção dos moluscos seguiu o protocolo de Deslandes; ${ }^{25}$ a identificação específica foi realizada de acordo com os protocolos estabelecidos por Paraense, ${ }^{26}$ com base nos caracteres morfológicos. A identificação molecular ${ }^{27}$ foi feita pelo Laboratório de Helmintologia e Malacologia Médica do Instituto René Rachou/Fiocruz, em pelo menos um exemplar de cada local de captura nos estados do Paraná, Minas Gerais e Pernambuco. Os perfis moleculares foram comparados aos perfis padrões do DNA extraído do tecido de moluscos da Coleção de Malacologia Médica (Fiocruz-CMM).

Para o tratamento dos dados, utilizou-se o software Stata versão 10.1 (Stata LP, College Station, TX, USA) e os mapas foram elaborados utilizando-se o software ArcGIS version 9.3 (Esri, Redlands, CA, USA).

As Secretarias de Estado da Saúde envolvidas apoiaram o estudo com a disponibilização de seus técnicos para atuação em campo e transporte até os locais de coleta. A formalização da anuência deu-se por meio de documento oficial.

A pesquisa foi realizada em conformidade com a Instrução Normativa $\mathrm{n}^{0} 141$, de 19 de dezembro de 2006, que regulamenta o controle e manejo ambiental da fauna sinantrópica nociva.

\section{Resultados}

Após levantamento realizado junto às coordenações estaduais e municipais, o número de municípios a serem trabalhados foi readequado, uma vez que 0 estado do Paraná já havia realizado o trabalho em 172 municípios. Para os demais estados, foram acrescidos

\section{Tabela 1 - Resultado da captura de Biomphalaria glabrata, Biomphalaria tenagophila e Biomphalaria straminea no} Paraná, Minas Gerais, Bahia, Pernambuco e Rio Grande do Norte, dezembro de 2012 - junho de 2014

\begin{tabular}{|c|c|c|c|c|c|c|c|c|c|c|}
\hline \multirow[b]{2}{*}{ Unidade Federada } & \multirow{2}{*}{$\begin{array}{l}\text { Municípios } \\
\text { pactuados }\end{array}$} & \multirow{2}{*}{$\begin{array}{l}\text { Municípios } \\
\text { acrescidos }\end{array}$} & \multirow{2}{*}{$\begin{array}{l}\text { Municípios } \\
\text { trabalhados }\end{array}$} & \multicolumn{2}{|c|}{$\begin{array}{c}\text { Municípios } \\
\text { pesquisados }\end{array}$} & \multicolumn{5}{|c|}{ Moluscos capturados, por espécie e municípios } \\
\hline & & & & $\begin{array}{l}\text { Total } \\
(\%)\end{array}$ & $\begin{array}{c}\text { Com } \\
\text { moluscos } \\
(\%)\end{array}$ & $\begin{array}{c}\text { B. } \\
\text { glabrata }\end{array}$ & $\begin{array}{c}\text { B. } \\
\text { tenagophila }\end{array}$ & $\begin{array}{c}\text { B. } \\
\text { straminea }\end{array}$ & $\begin{array}{c}\text { B. glabrata } \\
+ \\
\text { B. straminea }\end{array}$ & $\begin{array}{c}\text { B. glabrata } \\
+ \\
\text { B.tenagophila }\end{array}$ \\
\hline Paraná & 250 & - & $78^{a}$ & $78(100)$ & $18(23)$ & - & 2 & 16 & - & - \\
\hline Minas Gerais & 68 & 52 & 120 & $120(100)$ & $79(66)$ & 33 & 1 & 28 & 16 & 1 \\
\hline Bahia & 76 & 6 & 82 & $82(100)$ & $74(90)$ & $28^{b}$ & - & 14 & 32 & - \\
\hline Pernambuco & 19 & 32 & 51 & $51(100)$ & $40(78)$ & - & - & 38 & 2 & - \\
\hline Rio Grande do Norte & 95 & 3 & $98^{c}$ & $96(98)$ & $89(93)$ & 1 & - & 85 & 3 & - \\
\hline Total & 508 & 93 & 429 & $427(99)$ & $300(70)$ & 62 & 3 & 181 & 53 & 1 \\
\hline
\end{tabular}

a) Quando o projeto foi iniciado, a Secretaria de Estado da Saúde do Paraná já havia pesquisado 172 municípios.

b) Foram encontrados 18 exemplares positivos para S. mansoni nos seguintes municípios: Itororó (oito exemplares), Arataca (um) e Teolândia (nove).

c) Em dois municípios, não foram realizadas capturas em função da seca. 
93 municípios, sendo 52 em Minas Gerais, seis na Bahia, 32 em Pernambuco e três no Rio Grande do Norte. Neste último estado, não foi possível realizar a coleta em dois municípios (Grossos e Galinhos) em decorrência da mais severa estiagem dos últimos 100 anos e consequente ausência de ambientes favoráveis aos moluscos límnicos.

Dos 429 municípios selecionados para o estudo, foram obtidos dados de 427 municípios (99,5\%) (Tabela 1), sendo que em 300 (70,2\%) municípios foram encontrados moluscos do gênero Biomphalaria. Estes moluscos foram encontrados em 120 municípios no estado de Minas Gerais e 78 municípios do Paraná (Figura 1), na região Sudeste, e em 82 municípios da Bahia, 51 de Pernambuco e 98 do Rio Grande do Norte, na região Nordeste (Figura 2), dos quais 61 possuíam dados no acervo da coleção de moluscos do Instituto Oswaldo Cruz /(Fiocruz/CMIOC). A presença de $B$. glabrata foi registrada em 62 municípios, $B$. straminea em 181 e $B$. tenagophila em três municípios. Em 53 municípios, B. glabrata estava associada a B. straminea; e em um município, B. glabrata foi encontrada associada a $B$. tenagophila (Tabela 1 ).

Foram registrados moluscos infectados por $S$. mansoni em três (1\%) dos 300 municípios com presença de moluscos, todos no estado da Bahia: Itororó (oito exemplares), Arataca (um) e Teolândia (nove). Os resultados das coletas por município, nos cinco estados pesquisados (Figura 1A), são apresentados a seguir:

\section{Minas Gerais (Figura 1B)}

Biomphalaria glabrata (33): Aricanduva, Cachoeira de Pajeú, Capelinha, Carmésia, Catas Altas, Crisólita, Cristiano Otoni, Diogo de Vasconcelos, Divisópolis, Frei Gaspar, Itaverava, Jeceaba, Lamim, Mato Verde, Monte Formoso, Ouro Verde de Minas, Palmópolis, Piranga, Presidente Bernardes, Queluzita, Rio do Prado, Rio Espera, Rubim, Santa Helena de Minas, Santana dos Montes, Santo Antônio do Itambé, São Brás do Suaçuí, São Gonçalo do Rio Preto, Senhora de Oliveira, Senhora do Porto, Senhora dos Remédios, Serra Azul de Minas e Serro.

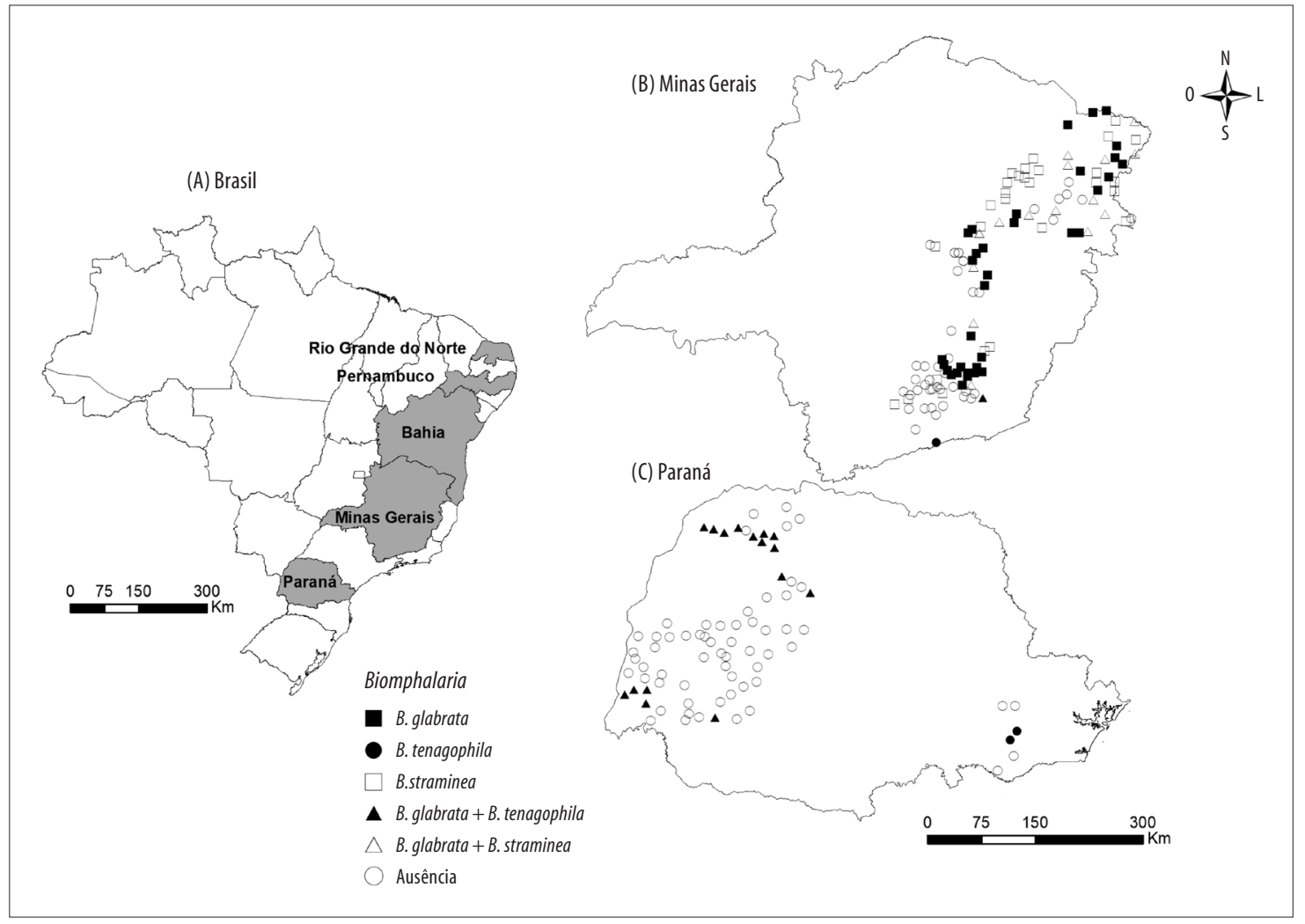

Figura 1 - Mapa do Brasil com destaque para os estados pesquisados (A) e distribuição dos hospedeiros intermediários do Schistosoma mansoni em Minas Gerais (B) e Paraná (C), dezembro de 2012 - junho de 2014 


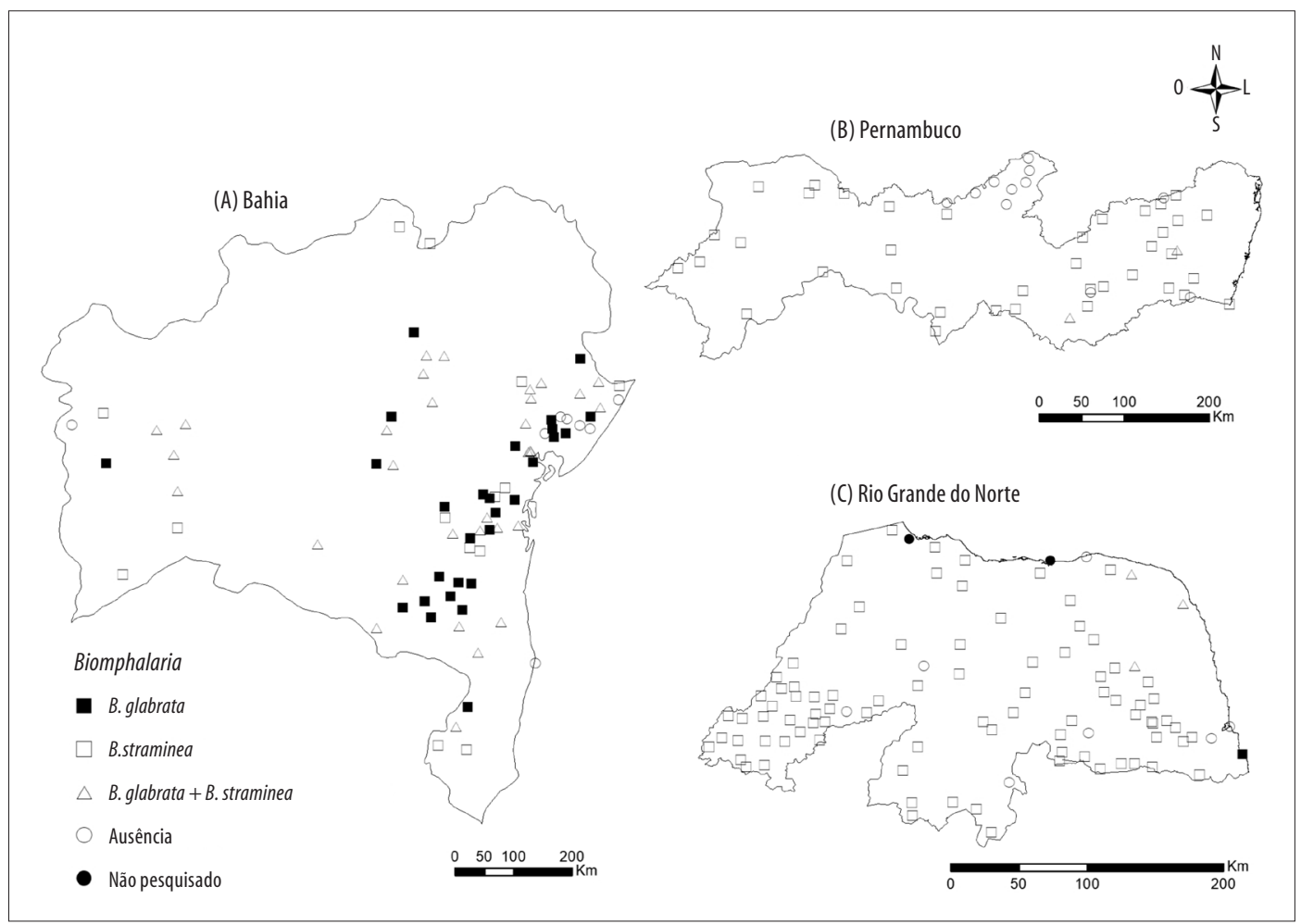

Figura 2 - Distribuição dos hospedeiros intermediários do Schistosoma mansoni na Bahia (A), Pernambuco (B) e Rio Grande do Norte (C), dezembro de 2012 - junho de 2014

Biomphalaria tenagophila (um): Santa Rita de Jacutinga.

Biomphalaria straminea (28): Acaiaca, Águas Formosas, Almenara, Araçuaí, Bandeira, Barra Longa, Barroso, Berilo, Bertópolis, Carbonita, Coronel Murta, Francisco Badaró, Franciscópolis, Fronteira dos Vales, Ingaí, Itutinga, Jenipapo de Minas, José Gonçalves de Minas, Lagoa Dourada, Leme do Prado, Monjolos, Nanuque, Santa Maria do Salto, Senador Modestino Gonçalves, Turmalina, Veredinha, Virgem da Lapa e Umburatiba.

B. glabrata/B. straminea (16): Alto Rio Doce, Alvorada de Minas, Ataléia, Carlos Chagas, Couto Magalhães de Minas, Felício dos Santos, Felisburgo, Itamarandiba, Itaobim, Jordânia, Ladainha, Pavão, Ponto dos Volantes, Santo Antônio do Jacinto, São Gonçalo do Rio Abaixo e Setubinha.

B. glabrata/B. tenagophila (um): Rio Pomba.

Municípios negativos (41): Angelândia, Carrancas, Casa Grande, Catuji, Conceição da Barra de Minas, Congonhas, Congonhas do Norte, Datas, Desterro de Entre Rios, Desterro do Melo, Dores de Campos, Entre Rios de Minas, Gouvea, Ibertioga, Ibituruna, Itaipé, Madre de Deus de Minas, Mercês, Morro do Pilar, Nazareno, Novo Oriente de Minas, Padre Paraíso, Paiva, Passa Tempo, Piedade do Rio Grande, Poté, Prados, Presidente Kubitschek, Raposos, Resende Costa, Ressaquinha, Ritápolis, Santa Bárbara do Tugúrio, Santa Cruz de Minas, Santana do Garambéu, Santo Antônio do Rio Abaixo, Santo Hipólito, São Tiago, Serra dos Aimorés, Serranos e Tiradentes.

\section{Paraná (Figura 1C)}

Biomphalaria tenagophila (dois): Araucária e Contenda.

Biomphalaria straminea (16): Fênix, Itaipulândia, Medianeira, Mirador, Missal, Nova Aliança do Ivaí, Paraíso do Norte, Planaltina do Paraná, Ramilândia, Santa Cruz de Monte Castelo, Santa Isabel do Ivaí, Santa Mônica, São Carlos do Ivaí, Tamboara, Terra Boa e Três Barras do Paraná.

Municípios negativos (60): Altamira do Paraná, Alto Paraná, Amaporã, Anahy, Araruna, Assis Chateaubriand, 
Boa Esperança, Boa Vista da Aparecida, Braganey, Cafelândia, Campina da Lagoa, Campo Bonito, Campo Largo, Campo Magro, Capitão Leônidas Marques, Catanduvas, Cruzeiro do Sul, Diamante D'Oeste, Diamante do Sul, Engenheiro Beltrão, Entre Rios do Oeste, Espigão Alto do Iguaçu, Formosa do Oeste, Guairaçá, Guaraniaçu, Ibema, Iguatu, Iracema do Oeste, Iretama, Janiópolis, Jesuítas, Lindoeste, Luiziana, Mamborê, Maripá, Matelândia, Mercedes, Nova Aurora, Nova Cantu, Nova Santa Rosa, Palotina, Pato Bragado, Peabiru, Quarto Centenário, Quatro Pontes, Quedas do Iguaçu, Quinta do Sol, Quitandinha, Rancho Alegre D'Oeste, Rio Negro, Roncador, Santa Helena, Santa Lúcia, Santa Tereza do Oeste, São João do Caiuá, São José das Palmeiras, São Pedro do Iguaçu, Serranópolis do Iguaçu, Ubiratã e Vera Cruz do Oeste.

\section{Bahia (Figura 2A)}

Biomphalaria glabrata (28): Andaraí, Barra do Choça, Cabaceiras do Paraguaçu, Catu, Entre Rios, Guaratinga, Ibicuí, Iguaí, Itambé, Itapicuru, Itororó, Jiquiriçá, Jitaúna, Lajedo do Tabocal, Maragogipe, Mirangaba, Nova Canaã, Nova Ibiá, Pedrão, Poções, São Desidério, Teodoro Sampaio, Teolândia, Terra Nova, Ubaíra, Utinga, Valença e Vitória da Conquista.

Biomphalaria straminea (14): Aiquara, Barreiras, Barrocas, Casa Nova, Cocos, Coribe, Ipuaú, Itanhém, Jandaira, Juazeiro, Lafaiete Coutinho, Laje, Mutuípe e Vereda.

B. glabrata/B. straminea (32): Anagé, Aporá, Apuarema, Arataca, Baianópolis, Biritinga, Cachoeira, Caem, Cândido Sales, Catolândia, Crisópolis, Esplanada, Feira de Santana, Gandu, Itapetininga, Ituberá, Jacobina, Jequié, Jucuruçu, Lagoa Real, Lamarão, Miguel Calmon, Mundo Novo, Muritiba, Nova Redenção, Potiraguá, Rio Real, Santa Maria da Vitória, São Felix, Serrinha, Wagner e Wenceslau Guimarães.

Municípios negativos (oito): Alagoinhas, Araçás, Aramari, Belmonte, Conceição do Jacuípe, Conde, Itanagra e Luiz Eduardo Magalhães.

\section{Pernambuco (Figura 2B)}

Biomphalaria straminea (38): Afrânio, Alagoinha, Calumbi, Camocim de São Félix, Capoeiras, Carnaubeira da Penha, Caruaru, Casinhas, Cedro, Cumaru, Dormentes, Feira Nova, Granito, Ibirajuba, Ipubi, Itacuruba, Itaiba, Jaqueira, Jataúba, Jatobá, Joaquim Nabuco, Jucati, Lagoa Grande, Manari, Moreilândia,
Orocó, Paranatama, Petrolândia, Poção, Riacho das Almas, Santa Cruz, Santa Filomena, Santa Maria Cambucá, São José da Coroa Grande, São José do Belmonte, Taquaritinga do Norte, Tupanatinga e Xexéu.

B. glabrata/B. straminea (dois): Iati e Saire.

Municípios negativos (11): Brejinho, Caetés, Iguaraci, Ingazeira, Quixaba, Santa Cruz da Baixa Verde, Santa Terezinha, São José do Egito, Solidão, Tuparetama e Vertente do Lério.

\section{Rio Grande do Norte (Figura 2C)}

Biomphalaria glabrata (um): Baia Formosa

Biomphalaria straminea (85): Afonso Bezerra, Almino Afonso, Antônio Martins, Areia Branca, Baraúnas, Barcelona, Boa Saúde, Bodó, Bom Jesus, Campo Redondo, Carnaúbas, Coronel Ezequiel, Coronel João Pessoa, Doutor Severiano, Encanto, Equador, Felipe Guerra, Fernando Pedrosa, Francisco Dantas, Frutuoso Gomes, Governador Dix-Sept Rosado, Guamaré, Ipueira, Itajá, Jaçanã, Jandaíra, Januário Cicco, Japi, Jardim de Angicos, João Dias, Judiá, Janduís, Lagoa de Pedras, Lagoa de Velhos, Lagoa Nova, Lagoa Salgada, Lajes, Lajes Pintadas, Lucrécia, Major Sales, Marcelino Vieira, Messias Targino, Montanhas, Monte das Gameleiras, Olho-d'Água dos Borges, Ouro Branco, Paraná, Passa e Fica, Passagem, Pau dos Ferros, Pedra Grande, Pedra Preta, Pilões, Porto do Mangue, Rafael Godeiro, Riacho da Cruz, Riacho de Santana, Riachuelo, Rodolfo Fernandes, Ruy Barbosa, Santana do Seridó, São Bento do Trairi, São Fernando, São Francisco do Oeste, São João do Sabugi, São Pedro, São Rafael, São Vicente, Senador Eloi de Souza, Serra Caiada, Serra de São Bento, Serra do Mel, Serrinha, Serrinha dos Pintos, Severiano Melo, Taboleiro Grande, Tenente Ananias, Tenente Laurentino, Tibau, Timbaúba dos Batistas, Triunfo Potiguar, Umarizal, Upanema, Venha-Ver e Viçosa.

B. glabrata/B. straminea (3): Rio do Fogo, Santa Maria e São Miguel do Gostoso.

Municípios negativos (sete): Caiçara do Norte, Carnaúba dos Dantas, Goianinha, Paraú, Patu, Santa Cruz e Tibau do Sul.

\section{Discussão}

Os resultados deste estudo mostram que a presença dos hospedeiros intermediários foi registrada em 70,2\% dos municípios estudados. 
Os dados levantados adicionam informações - até então escassas - aos arquivos das equipes de vigilância em saúde (http://tabnet.datasus.gov.br/ cgi/tabcgi.exe?sinan/pce/cnv/pcebr.def). Entretanto, os registros do presente trabalho estão de acordo com a distribuição conhecida para as três espécies hospedeiras intermediárias. ${ }^{9}$ É importante ressaltar a ocorrência simultânea de duas espécies hospedeiras intermediárias do $S$. mansoni, $B$ straminea e $B$. glabrata, em 53 municípios dos cinco estados sob análise. Para o Rio Grande do Norte, $62 \%$ das informações foram obtidas do acervo da coleção de moluscos do Instituto Oswaldo Cruz, reforçando a importância da política de preservação e manutenção das coleções biológicas da Fiocruz. Por outro lado, moluscos (B. glabrata) infectados por $S$. mansoni foram encontrados em apenas três municípios entre os 427 pesquisados. Esta pesquisa confirma: a distribuição geográfica desses moluscos vem sendo lenta, progressivamente acrescida de novas localidades, e corrobora os dados obtidos por inúmeros pesquisadores, a que se somam aqueles obtidos pela Fundação Nacional de Saúde, Secretarias de Estado da Saúde e Secretaria de Vigilância em Saúde/Ministério da Saúde, disponibilizados pelas Gerências do Programa de Vigilância e Controle da Esquistossomose. Esses bancos de dados foram alimentados por numerosas fontes e publicações. ${ }^{6-24}$

Em que pesem os esforços desenvolvidos, os dados sobre a real distribuição geográfica desses moluscos em todo o território nacional estão condicionados tanto à dificuldade de acesso a determinadas regiões do país quanto à falta de recursos financeiros e limitado número de pesquisadores atuantes nessa área específica. Outra limitação preponderante reside na capacidade de adaptação dos moluscos hospedeiros intermediários de $S$. manso$n i$ a condições ambientais impróprias, como a seca dos biótopos, impondo a necessidade da realização de buscas periódicas nos biótopos límnicos. ${ }^{28}$ Esses moluscos conseguem se enterrar, à medida que 0 ambiente seca; após as chuvas, repovoam o biótopo rapidamente, dada sua grande capacidade reprodutiva. Segundo Paraense ${ }^{28}$ um único exemplar de B. glabrata é capaz de produzir, cumulativamente, dez milhões de descendentes em três meses. Além do enterramento, esses moluscos podem sobreviver mesmo se infectados por $S$. mansoni, reduzindo seu metabolismo e permanecendo em diapausa ou anidrobiose ${ }^{29} \mathrm{~A}$ distribuição geográfica dos hospedeiros intermediários de $S$. mansoni no Brasil confere à esquistossomose um caráter expansivo, inclusive sobre as áreas consideradas indenes. ${ }^{6,9,30}$

A distribuição geográfica e a correta identificação das três espécies hospedeiras intermediárias do $S$. mansoni são importantes para orientar os agentes de saúde e as medidas de vigilância e controle ambiental em cada localidade, possibilitando a interrupção da cadeia epidemiológica da doença. Pesquisas como esta contribuem com os serviços de saúde no aprimoramento ou estruturação mais adequada das atividades de controle e vigilância da esquistossomose, direcionando ações para áreas de risco e racionalizando a aplicação dos recursos disponíveis. As atividades de monitoramento das populações dos moluscos hospedeiros intermediários do $S$. mansoni e a pesquisa de formas larvais de $S$. mansoni devem constituir atividades permanentes, enquanto medidas preventivas à expansão ou à instalação de focos de esquistossomose no Brasil não forem implementadas.

\section{Agradecimentos}

Às Secretarias de Estado da Saúde do Paraná, Minas Gerais, Bahia, Pernambuco e Rio Grande do Norte, pelo apoio logístico à realização deste trabalho.

A José Geraldo Amorim da Silva, do Laboratório de Helmintologia e Malacologia Médica do Instituto René Rachou/Fundação Oswaldo Cruz, pelo apoio técnico.

\section{Contribuição dos autores}

Carvalho OS, Thiengo SC, Caldeira RL, Fernandez MA e Marcelino JMR contribuíram substancialmente para a concepção, análise e interpretação dos dados, elaboração das versões preliminares e redação final do manuscrito. Scholte RGC contribuiu com a interpretação dos resultados, elaboração das versões preliminares, revisão da versão final, tratamento dos dados e construção dos mapas. Jannotti-Passos LK, Mendonça CLF, Carmo EH, Mesquita SG e Leal RS participaram da análise e interpretação dos resultados, redação e revisão do manuscrito. Todos os autores aprovaram a versão final do manuscrito e declaram ser responsáveis por todos os aspectos do trabalho, garantindo sua precisão e integridade. 


\section{Referências}

1. Ministério da Saúde (BR). Secretaria de Vigilância em Saúde. Departamento de Vigilância Epidemiológica. Vigilância da Esquistossomose Mansoni: Diretrizes Técnicas. 4. ed. Brasília: Ministério da Saúde, 2014.

2. Paraense WL. Distribuição dos caramujos no Brasil. In: Reis FA, Faria I, Katz N, organizadores. Modernos Conhecimentos sobre Esquistossomose Mansônica. Belo Horizonte: Academia Mineira de Medicina; 1986; v. 1, p.117-128

3. Corrêa LR, Paraense WL. Susceptibility of Biomphalaria amazonica to infection with two strains of Schistosoma mansoni. Rev Inst Med Trop São Paulo. 1971;13:387-90.

4. Paraense WL, Corrêa LR. Susceptibility of Biomphalaria peregrina from Brazil and Ecuador to two strains of Schistosoma mansoni. Rev Inst Med Trop São Paulo. 1973; 15:127-30.

5. Teodoro TM, Jannotti-Passos LK, Carvalho OS, Caldeira RL. Occurrence of Biomphalaria cousini (Mollusca: Gastropoda) in Brazil and its susceptibility to Schistosoma mansoni (Platyhelminths: Trematoda). Mol Phyl Evol. 2010; 57:144-51.

6. Carvalho OS, Schotte RGC, Amaral RS, Dutra LV, Guerra MAM. Distribuição espacial de Biomphalaria Glabrata, B. Straminea e B. Tenagophilia hospedeiros intermediários de Schistosoma Mansoni no Brasil. In: Carvalho OS, Coelho PMZ, Lenzi HL, organizadores. Schistosoma Mansoni e esquistossomose: uma visão multidisciplinar. Rio de Janeiro: Fiocruz; 2008. p. 529-46.

7. Piza JT, Ramos AS. Os focos autóctones de esquistossomose no Estado de São Paulo. Arq Hig Saúde Pública. 1960; 25:261-71.

8. Schlemper Junior BR, Ferreira Neto JA, Thiago PTS, Bressan C, Amarante AR. Distribuição geográfica de Planorbídeos em Santa Catarina, Brasil. Rev Soc Bras Med Trop. 1996; 29:411-18.

9. Paraense WL. Planorbídeos hospedeiros intermediários do Schistosoma mansoni. In: Cunha AS, organizadores. Esquistossomose mansoni. São Paulo: Universidade de São Paulo; 1970.

10. Paraense WL. Fauna planorbídica do Brasil. In: Lacaz CS, Baruzzi RG \& Siqueira Junior W organizadores. Introdução à geografia médica do Brasil. São Paulo: Edgard Blücher, Universidade de São Paulo; 1972.

11. Paraense WL. The distribution of the molluscan vectors of schistosomiasis in the Americas. Brasília Médica. 1975a;11:11-14.
12. Carvalho OS, Nunes IM, Caldeira RL. First report of Biomphalaria glabrata in the state of Rio Grande do Sul, Brazil. Mem Inst Oswaldo Cruz. 1998; 93:39-40.

13. Paraense WL. The schistosome vectors in the Americas. Mem Inst Oswaldo Cruz. 2001; 96(suppl):7-16.

14. Souza CP, Caldeira RL, Drummond SC, Melo AL, Guimarães CT, Delza MS, Carvalho OS. Geographical Distribution of Biomphalaria Snails in the State of Minas Gerais, Brazil. Mem Inst Oswaldo Cruz. 2001; 96:293-302.

15. Teles HMS, Pereira PAC, Richinitti LMZ Distribuição de Biomphalaria (Gastropoda, Planorbidade) nos Estados do Rio Grande do Sul e Santa Catarina, Brasil. Rev Saúde Pública de São Paulo. 1991; 25:350-2.

16. Teles HMS. Distribuição geográfica das espécies dos caramujos transmissores de Schistosoma mansoni no Estado de São Paulo, Brasil. Rev Soc Bras de Med Trop. 2005; 38:426-32.

17. Thiengo SC, Fernandez MA, Boaventura MFF, Stortti MA. A survey of freshwater gastropods in the Serrana Mesoregion of the state of Rio de Janeiro, Brazil. Mem Inst Oswaldo Cruz. 1998; 93 (supl I):233-4.

18. Thiengo SC, Fernandez MA, Boaventura MFF, Grault CE, Silva HF, Mattos AC, Santos SB. Freshwater snails and schistosomiasis mansoni in the State of Rio de Janeiro, Brazil: I - Metropolitan mesoregion. Mem Inst Oswaldo Cruz. 2001; 96:177-84.

19. Thiengo SC, Fernandez MA, Boaventura MFF, Magalhães MG, Santos SB. Freshwater snails and Schistosomiasis mansoni in the state of Rio de Janeiro, Brazil: III - Baixadas Mesoregion. Mem Inst Oswaldo Cruz. 2002a; 97:43-6.

20. Thiengo SC, Fernandez MA, Boaventura MFF, Silva HF, Mattos AC, Santos SB. Freshwater snails and Schistosomiasis mansoni in the State of Rio de Janeiro, Brazil: II - Centro Fluminense Mesoregion. Mem Inst Oswaldo Cruz. 2002b (5); 97:621-6.

21. Thiengo SC Mattos AC, Boaventura MF, Fernandez MA. Freshwater snails and Schistosomiasis mansoni in the state of Rio de Janeiro, Brazil: IV - Sul Fluminense Mesoregion. Mem Inst Oswaldo Cruz. 2004; 99:275-80.

22. Thiengo SC, Mattos AC, Santos SB, Fernandez MA. Freshwater snails and schistosomiasis mansoni in the state of Rio de Janeiro, Brazil: VI - Noroeste Fluminense Mesoregion. Mem Inst Oswaldo Cruz. 2006; 101 (supl. I):239-45. 
23. Thiengo SC, Santos SB, Fernandez MA. Malacofauna límnica da área de influência do lago da usina hidrelétrica de Serra da Mesa, Goiás, Brasil. I. Estudo qualitativo. Rev Bras Zool. 2005; 22:867-74.

24. Ministério da Saúde (BR). Secretaria de Vigilância em Saúde. Vigilância e controle de moluscos de importância epidemiológica. Diretrizes técnicas: programa de Vigilância e Controle da Esquistossomose (PCE). Brasília: Ministério da Saúde; 2008.

25. Deslandes N. Técnica de dissecação e exame de planorbídeos. Rev Serv Espec Saúde Pública. 1950; 4:371-82.

26. Paraense WL. Estado atual da sistemática dos planorbídeos brasileiros. Arq Mus Nac. 1975b; 55:105-28.

\footnotetext{
Abstract

Objective: to describe the geographical distribution of intermediate hosts of Schistosoma mansoni in five Brazilian states. Methods: this was a descriptive crosssectional study; municipalities were selected in the states of Paraná (78), Minas Gerais (120), Bahia (82), Pernambuco (51), and Rio Grande do Norte (98), for the period 2012 to 2014; these municipalities were chosen because they did not have current records of the presence of snails vectores de S. mansoni. The molluscs were captured and taxonomically identified and examined for S. mansoni cercariae. Results: the work was carried out in 427 municipalities (99.5\% of the 429 selected); the presence of mollusks was registered in 300 (70.2\%) municipalities; Biomphalaria glabrata were found in 62 (21\%) municipalities, B. straminea in 181 (60\%), B. tenagophila in three (1\%); B. glabrata/B. straminea association was found in 53 municipalities (18\%) and B. glabrata/B. tenagophila association in one (0.3\%) municipality. Conclusion: B. glabrata, B. straminea and B. tenagophila distribution records obtained in this study are consistent with previously known distribution.

Keywords: Schistosomiasis; Biomphalaria; Disease Vectors; Geographic Mapping.
}

27. Vidigal T, Caldeira RL, Simpson AJG, Carvalho OS. Further studies on the molecular systematic of Biomphalaria snails from Brazil. Mem Inst Oswaldo Cruz. 2000; 95:57-66.

28. Paraense WL. Self and cross-fertilization in Australorbis glabratus. Mem Inst Oswaldo Cruz. 1955; 53:285-91.

29. Barbosa FS, Coelho MV. Ação de dessecação sobre as fases larvárias intracaramujo de Schistosoma mansoni em Australorbis glabratus. Pub Av Inst Aggeu Magalhães. 1953; 11:159-62.

30. Carvalho OS, Rocha RS, Massara CL, Katz N. Primeiros casos autóctones de esquistossomose mansoni em região do Noroeste do Estado de Minas Gerais. Rev Saúde Pública São Paulo. 1988; 22:237-9.

\section{Resumen}

Objetivo: describir la distribución geográfica de los hospedadores intermediarios de Schistosoma mansoni en cinco estados brasileños. Métodos: estudio epidemiológico transversal; el estudio fue realizado en municipios de los estados de Paraná (78), Minas Gerais (120), Babia (82), Pernambuco (51) y Rio Grande do Norte (98), entre 2012 y 2014; estos municipios fueron elegidos por no poseer registros actualizados de la presencia de caracoles vectores de S. mansoni; los moluscos fueron capturados, taxonómicamente identificados y examinados para la verificación de cercarias de S. mansoni. Resultados: los trabajos fueron realizados en 427 municipios (99,5\% de 429 municipios seleccionados); fue registrada presencia de moluscos en 300 (70,2\%) municípios; la presencia de Biomphalaria glabrata fue registrada en 62 (21\%) municipios, B. straminea en 181 (60\%) y B. tenagophila en três (1\%); se observó asociación de B. glabrata con B. straminea en 53 (18\%) y de B. glabrata con B. tenagophila en uno (0,3\%). Conclusión: los registros de Biomphalaria están de acuerdo con la distribución conocida.

Palabras-clave: Esquistosomiasis; Biomphalaria; Vectores de Enfermedades; Mapeo Geográfico.

Recebido em 04/10/2017

Aprovado em 25/05/2018 